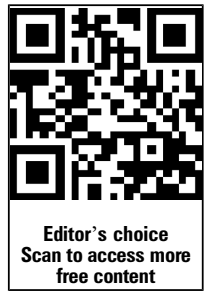

- Additional material is published online only. To view please visit the journal online (http://dx.doi.org/10.1136/bmjqs2012-001467)

${ }^{1}$ James M. Anderson Center for Health Systems Excellence, Cincinnati Children's Hospital Medical Center, Cincinnati,

Ohio, USA

${ }^{2}$ Division of Hospital Medicine, James M. Anderson Center for Health Systems Excellence, Cincinnati, Ohio, USA

${ }^{3}$ Department of Pediatrics, Cincinnati Children's Hospital Medical Center, Cincinnati, Ohio, USA

${ }^{4}$ Department of Management and Organizations, University of Michigan Ross School of Business, Ann Arbor, Michigan, USA

\section{Correspondence to} Linda M Goldenhar, James M. Anderson Center for Health Systems Excellence, Cincinnati Children's Hospital Medical Center, 3333 Burnett Ave ML 7140, Cincinnati, $\mathrm{OH} 45226$, USA;

linda.goldenhar@cchmc.org

Received 19 November 2012 Revised 29 April 2013 Accepted 17 May 2013 Published Online First 6 June 2013

\section{SLinked}

- http://dx.doi.org/10.1136/ bmjqs-2013-002322

To cite: Goldenhar LM, Brady PW, Sutcliffe KM, et al. BMJ Qual Saf 2013;22: 899-906.

\title{
Huddling for high reliability and situation awareness
}

\author{
Linda M Goldenhar, ${ }^{1}$ Patrick W Brady, ${ }^{2,3}$ Kathleen M Sutcliffe, ${ }^{4}$ \\ Stephen E Muething ${ }^{1}$
}

\begin{abstract}
Background Studies show that implementing huddles in healthcare can improve a variety of outcomes. Yet little is known about the mechanisms through which huddles exert their effects. To help remedy this gap, our study objectives were to explore hospital administrator and frontline staff perspectives on the benefits and challenges of implementing a tiered huddle system; and propose a model based on our findings depicting the mediating pathways through which implementing a huddle system may reduce patient harm.
\end{abstract}

Methods Using qualitative methods, we conducted semi-structured interviews and focus groups to obtain a deeper understanding of the huddle system and its outcomes as implemented in an academic tertiary care children's hospital with 539 inpatient beds. We recruited healthcare providers representing all levels using a snowball sampling technique (10 interviews), and emails, flyers, and paper invitations (six focus groups). We transcribed recordings and analysed the data using established techniques.

Results Five themes emerged and provided the foundational constructs of our model. Specifically we propose that huddle implementation leads to improved efficiencies and quality of information sharing, increased levels of accountability, empowerment, and sense of community, which together create a culture of collaboration and collegiality that increases the staff's quality of collective awareness and enhanced capacity for eliminating patient harm.

Conclusions While each construct in the proposed model is itself a beneficial outcome of implementing huddles, conceptualising the pathways by which they may work allows us to design ways to evaluate other huddle implementation efforts designed to help reduce failures and eliminate patient harm.

\section{BACKGROUND AND SIGNIFICANCE}

Huddles are not new to healthcare. These typically short briefings are designed to give frontline staff and bedside caregivers opportunities to stay informed, review events, make and share plans for ensuring well coordinated patient care.

Studies show that huddles can improve patient safety ${ }^{1-4}$ and can reveal factors that contribute to potentially adverse patient outcomes, such as medication errors, near misses and poor hand hygiene. ${ }^{5}$ They can provide a venue for raising concerns, increase efficiency of exchanging critical information, and increase staff's perception of the benefits of face-to-face discussion. ${ }^{6-8}$ Moreover, huddle implementation can improve teamwork by enhancing working relationships, increasing trust across departments, and helping staff appreciate and respect others, seeing them as allies working towards a common goal. ${ }^{2} 3$ 6-8

Missing from the literature is a description of how an integrated system of huddles, developed and structured based on theoretical principles, might work to reach the goal of reducing failures and eliminating patient harm. Thus, we conducted a qualitative study to begin addressing this gap. Specifically, our objectives were to describe the development and implementation of an inpatient huddle system, which was grounded in the theory of high-reliability organisations (HROs) and situation awareness (SA); explore the perspectives of hospital administrators and frontline staff on the benefits and challenges of huddle implementation; and use the findings to inform the development of a theoretical model depicting the pathways by which intermediate outcomes from huddle implementation may work together to increase staff's capacity to reduce patient harm.

\section{METHODS}

Guided by research findings showing the benefits of huddle implementation, Cincinnati Children's Hospital Medical 
Center (CCHMC), an academic tertiary care children's hospital with 539 inpatient beds, developed and began testing an inter-related tiered huddle system grounded in the theoretical principles of HROs and SA. ${ }^{9}$ The essential goals of implementing the huddle system were to improve the ability of staff and administration to identify emerging risks and threats, place those events in the proper organisational context, and formulate specific predictions and plans to efficiently and effectively resolve them.

\section{Huddle development and implementation: theoretical grounding}

Galvanised by the Institute of Medicine's landmark publication To Err is Human, ${ }^{10}$ healthcare researchers and improvers over the past decade have begun to investigate how healthcare can become more like other HROs. ${ }^{9}{ }^{10}$ HROs are defined by their ability to perform reliably and safely in the face of complexity and dynamism and include industries such as commercial and military aviation, the nuclear power industry and firefighting. ${ }^{9}$ While several HRO frameworks specific to healthcare have emerged, ${ }^{11}{ }^{12}$ transformation remains elusive, ${ }^{13}$ improvements remain modest and patient harm continues to be pervasive. ${ }^{14}$

Huddles are frequently used in HROs as a means for frontline staff to share and make sense of current situations, errors and concerns, and to discuss options for resolving or eliminating them in the future. Weick and Sutcliffe 9 outlined five key tenets of HROs: preoccupation with failure, avoidance of simplifying interpretations, sensitivity to operations, commitments to resilience (ie, capacity building), and flexible decision structures that defer to expertise. These were used to guide the design of CCHMC's inpatient inter-related huddle system (see figure 1). Specifically, preoccupation with failure is addressed by developing and implementing a required daily forum when staff review and reflect on all unexpected events; sensitivity to operations is achieved by continually assessing a unit's stress level and by bringing organisation resources and expertise to support unit functioning; reluctance to simplify interpretations is accomplished by ensuring that huddle participants represent a variety of disciplines and levels of experience so that multiple perspectives are considered when addressing issues; commitment to resilience is fostered by using proactive planning mechanisms to help detect and mitigate potential risky situations; and finally, deference to expertise is reflected by recognising that although unit staff closest to the work often have the best sense of what needs to be done, they sometimes face unusual or difficult circumstances that tax existing capabilities. During these times, units need to migrate and escalate decisions and actions to those with additional and different types of expertise.

In addition to the more general HRO tenets, the huddle system was designed to incorporate the principle of SA. A person displaying SA is able to monitor and recognise cues that increase their awareness of what is happening around them (perception); integrate information to develop a comprehensive picture of the current status and understand how it may affect goals (comprehension); and extrapolate forward to determine if the knowledge obtained might adversely influence the situation both immediately and in the near future (projection). ${ }^{15-17}$ Inadequate SA has been identified as a primary factor in accidents attributed to human error $^{17}$ and is especially critical in healthcare when information flow is high, continuous and complex, and poor decisions based on that information can lead to serious consequences. We

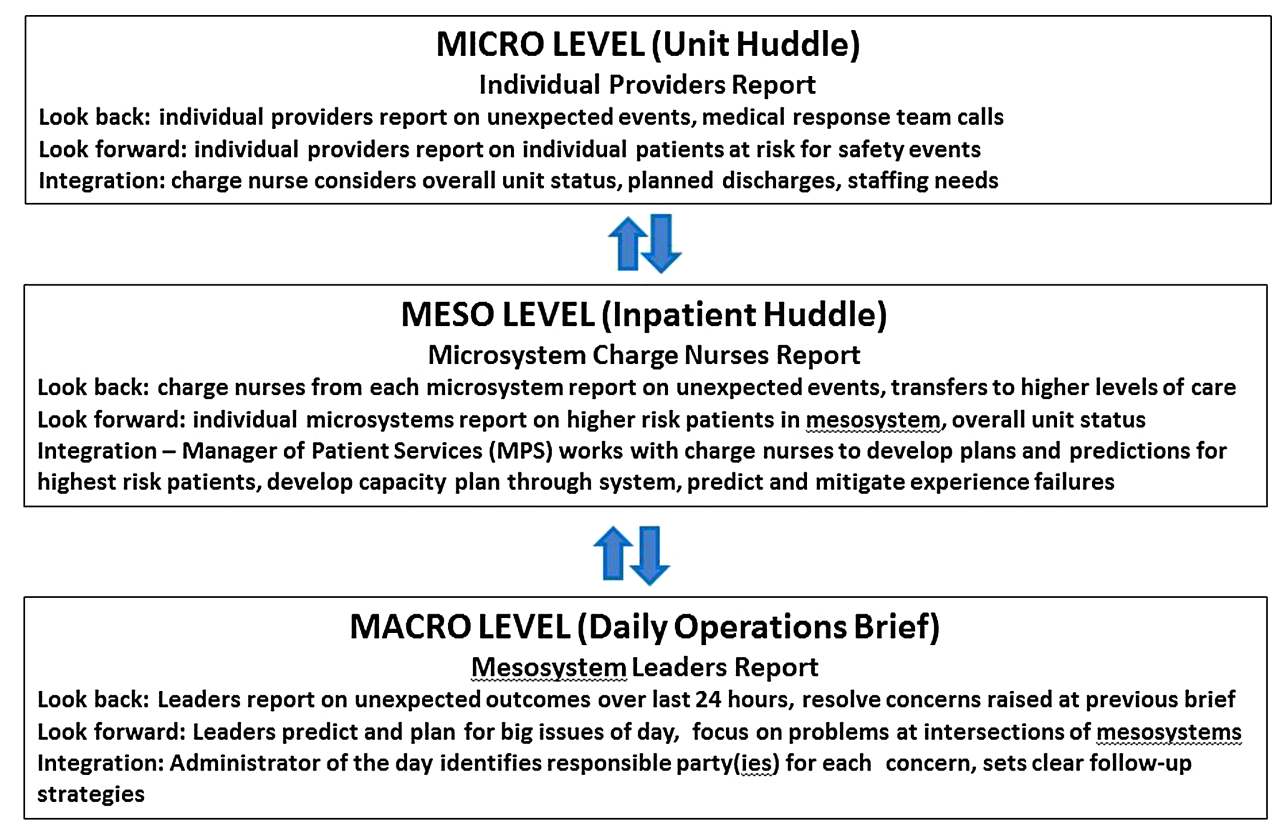

Figure 1 Three-level tiered huddle system grounded in high-reliability organisation-situation awareness principles. 
incorporated the SA principles into the huddle system by asking each huddle participant to systematically report on patients on their unit who they thought may deteriorate in the near future and label them as 'watchers' (perception); asking senior nurses and physician leads to coach charge nurses on how to integrate their perceptions into an informal severity of illness assessment (comprehension); and training the clinicians on how to use the information to facilitate prediction and planning for at-risk patients (projection). More detail on how SA was operationalised within the huddle system is reported in Brady et al. ${ }^{18}$

Table 1 shows a timeline of the development and implementation of the huddle system. The new HRO-SA based activities were initially incorporated into an already existing informal huddle process that was used to discuss flow and staffing issues. We conducted a pilot test in four units: general medical seeing patients $<12$ years old; general medical seeing adolescents; medical-surgical neurosciences; and short-stay surgery. Staff members participated in group training, one-on-one coaching, and peer role modelling on the new HRO-SA based activities and expectations. We developed standardised tools and a common lexicon, and instructed individuals at all levels to use them to collect data, conduct huddle reports, and develop mitigation and escalation plans. We conducted training in the remaining inpatient units approximately 5 months after the pilot projects were initiated. As shown in the data below, although it has taken time, the HRO-SA precepts introduced via the huddle system are now an integral part of day-to-day practices (see online supplementary appendix for more detail on huddle implementation).

\section{Study design}

We conducted a qualitative study, ${ }^{19}$ which allowed us to obtain a deeper and more comprehensive understanding of the huddle system and huddle outcomes from the participants' point of view. The study was deemed exempt by the CCHMC Institutional Review Board since it is part of an overall quality improvement effort and no identifying information was collected.

\section{Participants}

Interviews were conducted with a purposefully selected sample of key informants. In January 2012, the Vice President for Safety and the Director/ Manager of Patient Services (MPS) were recruited to participate as they were the champions of the new huddle effort. Then, using a snowball sampling technique ${ }^{20}$ they provided names of others who had been initially involved in developing and implementing the huddle system or were champions or current leaders in improving and expanding it. Those people identified additional staff members who were able to provide unique perspectives based on their organisational roles and huddle-related activities. All 10 individuals, representing diverse levels of the organisation, agreed to be interviewed (see box 1).

We also collected focus group data from inpatient bedside and charge nurses and respiratory therapists

Table 1 Cincinnati Children's Hospital Medical Center huddle development and implementation timeline

\begin{tabular}{|c|c|}
\hline Year & Huddle-related activities \\
\hline $\begin{array}{l}\text { 2005-2006 Pre } \\
\text { implementation }\end{array}$ & $\begin{array}{l}\text { Began speaking with people in other high-reliability organisations (HROs) } \\
\text { Concentrated learning about principles of HRO and situation awareness (SA) } \\
\text { Joined Agency for Healthcare Research and Quality HRO Learning Network } \\
\text { Initiated serious safety event (SSE) reduction across entire organisation }\end{array}$ \\
\hline $2007-2008$ & $\begin{array}{l}\text { Flow office began 8:00 bed huddles-focus on patient flow and staffing, run by Manager of Patient Services } \\
\text { Invited inpatient, emergency department (ED) and post-anaesthesia care unit directors_-changed to charge nurses (more frontline) } \\
\text { Started conducting data analysis of ED and community admissions } \\
\text { Attended Institute for Healthcare Improvement conference. Informed initial format of HRO and SA enhanced huddles and three-tiered } \\
\text { organisational huddle structure } \\
\text { Studied trend data and identified SA failures related to SSEs } \\
\text { Invited outside SA experts to consult }\end{array}$ \\
\hline 2009 & $\begin{array}{l}\text { Four diverse units piloted methods to improve SA; focused on improving perception and comprehension } \\
\text { Created safety officers of the day (SOD) role (started with one, expanded to four within } 1 \text { year) } \\
\text { Began developing and testing standardised data identification and collection tools }\end{array}$ \\
\hline 2011 & $\begin{array}{l}\text { Emphasised robust mitigation plans } \\
\text { Invited representatives from family relations, protective services, social services, and facilities to 8:00 bed huddle } \\
\text { Began tracking SA concerns in electronic health record }\end{array}$ \\
\hline 2012 & $\begin{array}{l}\text { Began midnight huddles } \\
\text { Began weekend daily operational brief } \\
\text { Began new pilot to enhance structure and standardisation of unit huddles }\end{array}$ \\
\hline
\end{tabular}




\section{Box 1 Interview participants, titles/hospital roles}

Vice President for Safety (also a safety officer) Paediatric Intensive Care Unit Medical Director (also a safety officer)

- Chief of Staff (also a safety officer)

- Senior VP Medical Operations, Department of Surgical Services, paediatric surgeon

- Two hospital medicine physicians (one is a safety officer)

- Senior Director of Patient Safety (a nurse)

- Director, Manager of Patient Services (MPS) (a nurse)

- Clinical manager/charge nurse on a general medical unit

- Patient flow coordinator (prior role as a charge nurse)

- Bedside nurse on a general medical unit

(RTs) who were participating in a separate qualitative study on SA (conducted by PB and LG). That study's aim was to assess perceptions of and experiences with SA more broadly; however participants often mentioned huddles during the focus group sessions. Thus, we included all huddle-related comments made by these key stakeholders in the qualitative dataset for this study.

To maximise focus group participation we sent emails and hard-copy invitations to all inpatient charge and bedside nurses and RTs $(\mathrm{N}=700)$ and also displayed flyers in high-visibility areas (eg, restrooms and break rooms). To encourage an honest and open dialogue, we assigned participants to a group based on their current role and responsibilities resulting in three charge nurse groups $(n=3 ; n=3 ; n=4)$ and three bedside nurse/RT groups $(\mathrm{n}=3 ; \mathrm{n}=3 ; \mathrm{n}=5)$. Participants represented 14 of the 19 inpatient units and included units caring for acute and critical care, general paediatric and subspecialty, and medical and surgical patients. The majority of participants had been on their current unit for $>5$ years and over half had $\geq 10$ years of nursing experience.

\section{Data collection}

Throughout February 2012, LG (an evaluator unaffiliated with the huddle activities) conducted hour-long, face-to-face interviews in each participant's office or another private location using a semi-structured interview guide. The interview was composed of openended questions to elicit perceptions of the purpose, structure, benefits and challenges of huddles, and was tailored for each interviewee type. LG conducted the focus groups in a conference room setting using a different semi-structured interview guide that included more general questions about SA. All interviews/focus groups were digitally recorded and transcribed verbatim.

\section{Analysis}

We analysed transcript data using the constant comparison approach ${ }^{21}$ to identify convergent and divergent perspectives on the purpose and goals of huddles, as well as their perceived benefits and challenges. LG and PB independently open coded a small subset of the interviews. They met to compare and discuss their results and to make final coding decisions which were used to create an initial codebook. They used that codebook to analyse subsequent transcripts, updating and revising it as additional codes emerged. Relevant training materials, presentations, data collection and reporting forms were also reviewed. Initial findings and the emerging model were shared with interviewees to assess validity and obtain feedback and additional context.

\section{FINDINGS}

\section{Beneficial outcomes}

Our analyses revealed five overarching themes related to the benefits of the tiered huddle system. We discuss each theme below along with illustrative quotes (additional quotes can be found in table 2).

Improved efficiencies and quality of information sharing

The outcome most often mentioned was that the new huddle structure facilitated more (quantity) and better (quality) information sharing and communication within and across participating units (micro) and mesosystems (ie, emergency department, perioperative, employee safety). Training and coaching on HRO and SA principles, and theory-based data collection and reporting tools, facilitated huddle participants' and leaders' use of a standardised, consistent language and terminology. This uniformity, they noted, enhanced their ability to communicate with each other and increased a shared understanding of the cultural assumptions of safety, risk and threats. A majority of interviewees also noted that having designated times each day for interacting with and listening to representatives from other interconnected units was critical for accomplishing well coordinated care. Some informants, particularly nurses and nurse leaders, opined that huddles provided a scheduled venue for asking questions and obtaining answers in real time rather than having to make multiple phone calls over many hours. These perceptions are well illustrated in the following quote:

\footnotetext{
We learned the new terminology ... We learned what a watcher was, we learned what high risk therapy was, and then in practice continued to report these concerns, we began to ... identify who was at risk on your unit, and who wasn't. (Bedside nurse)
}

\section{Accountability}

In addition to improvements in information and communication, participants repeatedly reported that the new huddle system demanded a greater degree of accountability for being able to verbalise concerns about 
Table 2 Additional illustrative quotes for each theme

\begin{tabular}{|c|c|}
\hline Theme & Illustrative quotes \\
\hline $\begin{array}{l}\text { Improved efficiencies and quality } \\
\text { of information sharing }\end{array}$ & $\begin{array}{l}\text { "... when you go to bed huddle, you're actually starting to hear the same language and people are talking about } \\
\text { everything in the same way ... Everyone's actually starting to embrace it as part of everyday [culture]" (bedside nurse) } \\
\text { "The main thing [is it] formalizes it at the organisational level in a tight communication. Don't assume all is ok just } \\
\text { because you don't hear about it" (safety officer of the day (SOD)) } \\
\text { "[It] establishes structure, culture, communication techniques that have reliable outcomes and also the process in place } \\
\text { to analyze why unexpected outcomes happened and try to prevent them from happening in the future" (SOD) }\end{array}$ \\
\hline Accountability & $\begin{array}{l}\text { "Accountability and the ability to escalate. Not only the ability, but the expectation that you will not proceed in the } \\
\text { face of uncertainty. If you are ... uncomfortable ... you are expected to call ... and the problem will be resolved" } \\
\text { (SOD) } \\
\text { "When you go to bed huddle and you see that all these other units are doing it and you're having to state these out } \\
\text { loud in front of everyone when you are discussing your unit, it puts the accountability on that individual. Even if they } \\
\text { didn't see it as a priority ... they have to do it and by doing it then I think people start to understand the importance } \\
\text { of it" (charge nurse) } \\
\text { "[The huddle] provides some peer-pressure for working on processes at the floor level to learn about how to talk about } \\
\text { what's happened in the last } 24 \text { hours and predictions for the next } 24 \text { (or less) hours" (charge nurse) }\end{array}$ \\
\hline Empowerment & $\begin{array}{l}\text { "Now there's more authority on the MPS side to bring the attendings together and work with them on the plan" } \\
\text { (Manager of Patient Services (MPS)) } \\
\text { "I'm challenged [now] to take it to the next level and ... you know something will be done about that problem" } \\
\text { (charge nurse) } \\
\text { "At times, the physicians don't agree on what approach to take. So, now the nurse can call in the MPS or the SOD } \\
\text { who will call the attendings' and say: 'we need to have a conference to discuss the situation and a clear plan on how } \\
\text { to proceed" (MPS) }\end{array}$ \\
\hline Sense of community & $\begin{array}{l}\text { "Many units knew what was going on in their own world, but nurses didn't know each other and had tunnel vision } \\
\text { about their needs" (Director, MPS) } \\
\text { "[Nurses at the huddle] felt they had a much better view of what was going on house-wide which helped them both } \\
\text { manage their own unit and feel connected to everyone else rather than like islands out on their own" (MPS) }\end{array}$ \\
\hline
\end{tabular}

current patient status and to effectively present mitigation and escalation plans to peers, supervisors, the safety officer of the day (SOD) and other attendees. Participants observed that being able to meet these new expectations and be more accountable gave them more authority in the eyes of their colleagues and team members. Increased accountability was noted as a beneficial huddle-related outcome by individuals at all levels:

One of things that it seems like this has done, this whole processes and structure increased accountability across the hospital really. (Chief of staff)

\section{Empowerment}

Charge and bedside nurses, in particular, reported that the new huddle structure helped them feel more empowered to speak up and publicly express disagreement, even with those in 'power'. They declared that they have a more credible voice at the individual, unit and organisational levels, and that this has led to a higher degree of trust from fellow providers, including physicians. This increased level of trust has resulted in their ideas and recommendations being taken seriously and acted on, rather than dismissed, leading them to feel empowered to continue providing ideas and take the initiative on new projects. This sentiment is expressed in the following quote:

We have come leaps and bounds here with nursing feeling like they are empowered to speak to and to push back on physicians a little bit which was not the culture, but we have grown, but there are still barriers, especially with certain senior level. (Charge nurse/clinical manager)
Sense of community

Participants at all levels reported that the huddle system has enabled a more comprehensive hospitalwide view of patient safety, census, staffing, admissions and discharges, and that this has led to a greater sense of community. Moreover, they stated that this larger vision gave them a much deeper understanding of what their colleagues across the hospital deal with on a daily basis, which makes them feel more connected to their peers, to other staff and to the organisation as a whole. One bedside nurse reflected how the increased sense of community is manifested at the individual level: 'It's easy to dismiss someone you don't know'. This view is further illustrated by the following quote:

... there is a greater sense of community between the charge nurses. When I was in charge, I was able to see the bigger picture of what was going on in the medical centre and not just what was happening on [my] particular unit. I was able to see [if] the census is very high so it's important to get our discharges out as quickly as we can. (Charge nurse)

Culture of collaboration/collegiality

The final theme that emerged was that the huddle system appears to promote a culture of increased staff collaboration and collegiality. Participants noted that there was less competition for beds and staff, that they were more likely to offer beds and staff to other units, and that more realistic requests for staff needs are made. In terms of safety, they said that during the huddles, participants were more likely to provide 
advice and consultation to nurses who work on non-specialised floors, but were asked to take care of children with specialised needs due to high census. This sentiment is expressed in the following quote:

Anti-competition, consideration, compassion-don't assume that the unit is saying no because they don't want to help, all have a better idea of what's going on on other units and know that everyone is busy!(MPS)

\section{Challenges}

Surprisingly, very few participants mentioned any huddle-related challenges. Only two, time and personnel resources, were mentioned by more than one interviewee; and focus group participants did not mention any. The concerns noted are reflected in the following comments:

They take time, they take you away from your primary place ... we're talking about in the actual physical huddle 20-30 people sitting around for the daily brief, I don't know how many people are on the phone.(SOD)

As a charge nurse ... sometimes you had a patient who was deteriorating on your unit and you didn't want to leave the patient at the bedside or you had a family need that you had to directly attend to. So sometimes you couldn't go. (Charge nurse)

\section{Toward a proposed model of huddle effects}

While the themes emerged independently from the data, in figure 2 we propose a model illustrating that the capacity to reduce failures and eliminate patient harm may result from a process whereby improved efficiencies and quality of information sharing, increased accountability, empowerment and a greater sense of community jointly enable a culture of collaboration and collegiality, ultimately resulting in an increased quality of collective awareness.

\section{DISCUSSION}

Studies within and outside of healthcare have shown that huddle implementation can result in and improve on a number of beneficial outcomes, including improved communication and collaboration. ${ }^{1-6} 8{ }^{8} 182223$ While our findings are consistent with these earlier studies, we are proposing that rather than considering each concept individually, it may be more useful to view them as mediating variables working together to improve care, eliminate failures and reduce patient harm. Specifically, the model suggests that huddle implementation can help systematise clinically related communication activities and provide a new lexicon for creating a shared meaning of more abstract ideas like patient safety and threat. This shared meaning can then result in improved efficiency of information sharing, a heightened sense of accountability and empowerment, and an increased sense of community across different units and groups. These factors together may then strengthen the organisation's collaborative culture for enhanced learning and understanding, which we could result in reduced failures and patient harm. ${ }^{24}$ Of course, the only way to test this model is to create a valid and reliable survey instrument measuring each of the constructs and administer it to a representative sample of healthcare providers prior to and after a new huddle system has been implemented. This will be the next step in our huddle-related research agenda.

A heightened sense of accountability was not simply manifested in increased responsibility for completing assignments or carrying out specific tasks. Rather, as we showed earlier, it had to do with a broader expectation that all staff members must assume more personal responsibility for ensuring that reliable identification, mitigation, and escalation of concerns occur. Accountability, the data suggest, was fuelled in two ways. First, through training and coaching all staff members in using standardised tools and improving their skills and capabilities using them. Second, by establishing expectations and processes that required staff members to use their skills to be conscious of patient-related cues as they emerge, to comprehend the possible adverse consequences of those cues, and make any needed adjustments to action before they turn into a serious safety event (SSE). ${ }^{25}$ These findings are consistent with other published studies describing the mechanisms through which operating room briefings result in operating room personnel taking increased responsibility for safety. ${ }^{2}$

The construct of empowerment has been defined in many ways, depending on the context in which it is

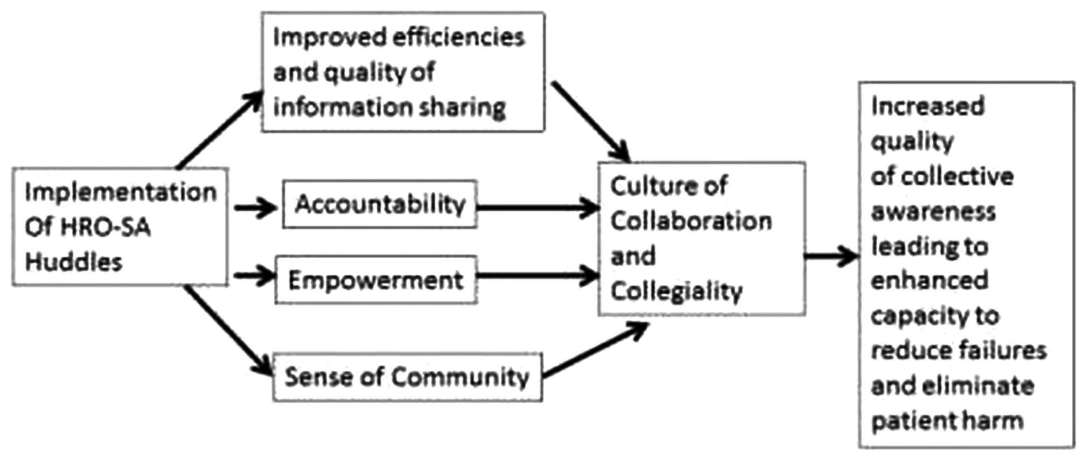

Figure 2 Proposed model of how emerging themes/concepts might work together to improve collective awareness, reduce failures and improve patient care. HRO, high-reliability organisation; SA, situation awareness. 
being studied and used. ${ }^{26}$ Our participants used it to describe how the huddle system and standardised SA language and tools provided nurses, in particular, the opportunity and confidence to speak up to other team members, supervisors or others of higher status when they disagree with a particular course of action. This finding is consistent with work on preoperative huddles showing that the opportunity for team members to seek input from the whole team participating in the huddle is associated with decreased rates of wrong-site surgeries. ${ }^{3} 22$

We imagine that empowerment, like sense of accountability, was enhanced by the coaching activities that were enacted as part of the huddle implementation efforts. Huddle leaders were able to identify anxious or less confident clinicians and work with them to increase their level of comfort and sense of empowerment to speak up and express concerns in the huddle environment and on the clinical floor. Empowered frontline clinicians are essential for improving individual and organisational SA and for ensuring that interpretations of complex events are not oversimplified, an important HRO tenet.

As our participants noted, huddles also stimulated an increased sense of community, a feeling of being connected to other teams and units at the meso and macro levels. Participants reported that they had a greater appreciation of their place and role in the organisation and that they felt more connected to their peers outside of their primary work area, both of which ultimately facilitated collaboration across units. The huddle system created a safe environment for presenting and discussing challenges and threats to patient safety that commonly exist throughout a healthcare organization, which is critical to facilitating a transition to an HRO culture more preoccupied with understanding and eliminating failure.

While an enhanced culture of collaboration and collegiality has been identified in the literature as an immediate outcome of the huddles, ${ }^{3}$ we suggest, as depicted in the proposed model, that the other proximal outcomes described earlier may be necessary and indeed work together to increase the likelihood of sustained collaboration and collegiality. As several participants noted, the huddles served to create a 'we are all in this together' mentality that facilitated smooth and efficient collaboration when, for example, a nurse needed advice on giving an unfamiliar medicine or to determine the best unit placement for a complex patient leaving the intensive care unit. This type of collaboration is essential for progressing toward an HRO culture of deference to expertise and commitment to resilience. Collaboration across disciplines and microsystems ensured varied frontline expertise was available and facilitated proactive planning.

Participants did not mention reducing SSEs as a positive outcome of huddle system implementation. While we cannot assert a causal link between huddles and reduction of SSEs, data from a recent quality improvement effort showed that, since huddle enactment, there has been a 50\% reduction in some SSEs, including unrecognised clinical deterioration. ${ }^{18} \quad 27$ One possible explanation for why participants did not mention SSEs is that safety is a 'non-event' until something adverse happens. ${ }^{28}$ That is, when asked about huddles, participants focused more on what they experience on a daily basis (proximal model outcomes) versus the rarer, more distal outcome of a SSE.

The study has several limitations. First, some may argue that the snowball sampling strategy for obtaining key informant interviewees could have resulted in a biased and more positive view of the HRO-SA huddle development and implementation process. While this may be possible, we believe that the diverse nature of the interviewees in terms of roles and responsibilities within the hospital helped attenuate the likelihood. It is true that we did not specifically seek out individuals who may have had negative impressions of the huddle system. Although participants noted many benefits, they also mentioned challenges. The low focus groups' participation rate of less than 3\% may indicate that only those with positive huddle experiences volunteered. However, the aims for the focus group study did not pertain to huddle development or implementation and therefore the recruitment materials and efforts did not specify the goal of learning about huddles. Despite this, selection bias may exist, resulting in the over-representation of positive themes and under-representation of negative themes. Additionally, a few of the interviewees participated in developing the huddle system, and two (PB and SM) are also members of our study team. Again, some may argue this resulted in positive bias. However, this could be countered by the fact that it is these individuals in particular who are aware of any challenges that need to be addressed. Another limitation is that we focused only on the inpatient huddle system which may be structurally or culturally different from other types of units within or outside our hospital Thus, it is uncertain how generalisable our proposed model will be to other departments and centres with different huddle implementation models, safety cultures, levels of physician and nursing engagement, and patient populations. These are all important considerations for future research efforts.

\section{CONCLUSIONS}

Hospital leadership determined that a system of interrelated huddles grounded in high-reliability theory could potentially reduce patient harm across the hospital. Once implemented, the system provided systematic and regular opportunities for micro, meso and macrosystem level leaders and others to interact face to face to examine and solve problems together. As our findings suggest and our model proposes, in addition 
to the more concrete act of solving problems, huddle implementation appears to improve the efficiency of information sharing among staff, enhance their sense of accountability and empowerment, and strengthen their sense of community, which, as we suggest in the model, may work together to establish a more collaborative culture that enables a collective awareness for reducing failures and improving patient safety.

Acknowledgements We want to thank Beth Mainwaring for her participation in reviewing the literature for this study. We also want to thank all the nurses and physicians who took time out of their busy schedule to speak with us about the new huddle system. We learned a lot and look forward to using their ideas to make it even better.

Contributors LMG: provided initial conceptualisation of the study and study design, conducted data collection, analysis, and interpretation, drafted the article, critically reviewed and revised it, and initially conceived the model. PWB: participated in conception and study design, analysis and interpretation of data, drafting the article, critically reviewing, and revising it for important intellectual content and final approval of the version to be published and was one of the key informants. KMS: participated in the conception and design, interpretation of data, critically revising initial drafts of the article, and providing important intellectual content and final approval of the version to be published. SEM: participated in interview as key

informant, helped draft historical and implementation aspects of the article, reviewed it for important intellectual content and provided final approval of the version to be published.

\section{Competing interests None.}

Ethics approval Institutional Review Board.

Provenance and peer review Not commissioned; externally peer reviewed.

Data sharing statement We have additional quotes in a database. These are available on request from the corresponding author.

\section{REFERENCES}

1 Edelson DP, Litzinger B, Arora V, et al. Improving in-hospital cardiac arrest process and outcomes with performance debriefing. Arch Intern Med 2008;168:1063-9.

2 Leonard M, Graham S, Bonacum D. The human factor: the critical importance of effective teamwork and communication in providing safe care. Qual Saf Health Care 2004;13(Suppl 1): i85-90.

3 Makary M, Mukherjee A, Sexton J, et al. Operating room briefings and wrong-site surgery. J Am Coll Surg 2007;204:236-43.

4 Paull DE, Mazzia LM, Wood SD, et al. Briefing guide study: preoperative briefing and postoperative debriefing checklists in the Veterans Health Administration medical team training program. Am J Surg 2010;200:620-3.

5 Wilbur K, Scarborough K. Medication safety huddles: teaming up to improve patient safety. Can J Hosp Pharm 2005;58:151-5.

6 Cooper R, Meara M. The organizational huddle processoptimum results through collaboration. Health Care Manag (Frederick) 2002;21:12-16.

7 Dingley C, Daugherty K, Derieg MK, et al. Improving patient safety through provider communication strategy enhancements.
In: Henriksen K, Battles JB, Keyes MA, et al. eds. Advances in patient safety: new directions and alternative approaches (Vol. 3: Performance and Tools). Rockville, MD: Agency for Healthcare Research and Quality (US), 2008.

8 Marks LB, Jackson M, Xie L, et al. The challenge of maximizing safety in radiation oncology. Pract Radiat Oncol 2011;1:2-14.

9 Weick K, Sutcliffe K. Managing the unexpected: resilient performance in an age of uncertainty. 2nd edn. San Francisco: Jossey-Bass, 2007.

10 Kohn LT, Corrigan J, Donaldson MS. To err is human: building a safer health system. Washington, DC: National Academy Press, 2000.

11 Chassin M, Loeb J. The ongoing quality improvement journey: next stop, high reliability. Health Aff (Millwood) 2011;30:559-68.

12 Pronovost P, Berenholtz S, Goeschel C, et al. Creating high reliability in health care organizations. Health Serv Res 2006;41(4 Pt 2):1599-617.

13 Roberts KH, Madsen P, Desai V, et al. A case of the birth and death of a high reliability healthcare organisation. Qual Saf Health Care 2005;14:216-20.

14 Landrigan C, Parry G, Bones C, et al. Temporal trends in rates of patient harm resulting from medical care. $\mathrm{N} \mathrm{Engl} \mathrm{J} \mathrm{Med}$ 2010;363:2124-34.

15 Endsley M. Measurement of situation awareness in dynamic systems. Hum Factors 1995;37:65-84.

16 Endsley M. Toward a theory of situation awareness in dynamic systems. Hum Factors 1995;37:32-64.

17 Endsley M, Garland D. Situation awareness: analysis and measurement. Mahwah, NJ: Lawrence Erlbaum Associates, 2000.

18 Brady PW, Muething S, Kotagal U, et al. Improving situation awareness to reduce unrecognized clinical deterioration and serious safety events. Pediatrics 2013;131: e298-308.

19 Flyvbjerg B. Case study. In: Lincoln NKDaYS, ed. The Sage handbook of qualitative research. Thousand Oaks, CA: Sage, 2011:301-16.

20 http://www.nsf.gov/bfa/dias/policy/hsfaqs.jsp\#snow. The National Science Foundation, 2008.

21 Patton M. Qualitative evaluation and research methods. 2nd edn. Thousand Oaks, CA: Sage Publications, 1990.

22 Khoshbin A, Lingard L, Wright JG. Evaluation of preoperative and perioperative operating room briefings at the Hospital for Sick Children. Can J Surg 2009;52:309-15.

23 Setaro J, Connolly M. Safety huddles in the PACU: when a patient self-medicates. J Perianesth Nurs 2011;26:96-102.

24 Vogus TJ, Sutcliffe KM, Weick KE. Doing no harm: enabling, enacting, and elaborating a culture of safety in health care. Acad Manag Perspect 2010:60-77.

25 Perin C. Shouldering risks: the culture of control in the nuclear power industry. Princeton, NJ: Princeton University Press, 2006.

26 Honold L. A review of the literature on employee empowerment. Empowerment Organ 1997;5:202-12.

27 SE M, A G, PJ S, et al. Quality improvement initiative to reduce serious safety events and improve patient safety culture. Pediatrics 2012;130:423-31.

28 Weick KE. Organizational culture as a source of high-reliability. Calif Manage Rev 1987;29:112-27. 